\title{
Campaign for IT literacy through FOSS and Spoken Tutorials
}

\author{
Kannan M. Moudgalya ${ }^{\ddagger *}$
}

\begin{abstract}
This article explains an approach to promote Information Technology (IT) literacy in India, which has evolved into a pyramid structure. We begin this article by explaining the design decisions, such as the use of FOSS and being a friendly interface between beginners and experts, in undertaking this activity.

A Spoken Tutorial is a ten minute audio video tutorial on open source software, created to provide training on important IT topics. Spoken Tutorials are made suitable for self learning, through a novice check of the underlying script. The spoken part of these tutorials is dubbed in all Indian languages, to help children who are weak in English, while retaining employment potential. The effectiveness of conducting workshops using spoken tutorials is explained. A total of 400,000 students have been trained in the past three years through one or more Spoken Tutorial based Education and Learning through Free FOSS study (SELF) workshops.
\end{abstract}

Students who undergo SELF workshops can create textbook companions, which contain code for the solved problems of given textbooks using a particular software. A Python Textbook Companion is a collection of Python code for a given textbook. These companions and the associated textbook, together, form a low cost document for Python in general, and the textbook, in particular. We have completed approximately 80 Python Textbook Companions and another 80 are in progress. From textbook companions, the students can progress to lab migration activity, the objective of which is to migrate labs based on proprietary software to FOSS. Interested students are trained to use FOSS systems in their projects and to contribute to the development of new FOSS systems. Using this approach and Python as a glue language, we have developed the following new FOSS systems: 1. Oscad, an electronic design automation tool, and a FOSS alternative to commercial software, such as ORCAD. 2. Sandhi, a block diagram based data acquisition for real time control, and a FOSS alternative to commercial software, such as LabVIEW.

The pyramid structure explained in this work helps the beginners to become IT literate. Our design solutions are especially suitable to poor and marginalised sections of the society, which is at the bottom of the social pyramid. Our efforts to create and promote the world's lowest cost computing system Aakash is briefly addressed in this work.

Index Terms-Python, spoken tutorials, FOSSEE

\section{Introduction}

This article explains the approach we have taken to promote IT literacy in India. While India has the capability to create software to help improve the lives of people around the world, its citizens do not have the capability to absorb it. One main reason is that India does not have a good infrastructure. In addition, the economic

* Corresponding author: kannan@iitb.ac.in

* Dept. of Chemical Engineering, Education Technology, and System and Control Groups, IIT Bombay, India

Copyright $\odot 2014$ Kannan M. Moudgalya. This is an open-access article distributed under the terms of the Creative Commons Attribution License, which permits unrestricted use, distribution, and reproduction in any medium, provided the original author and source are credited. conditions do not allow our citizens in general, and students in particular, to buy expensive hardware and software. Lack of good quality educational institutions and good teachers compound the problem.

This paper begins with the design decisions we have taken to achieve our objective while keeping in mind the problems mentioned above. Some of the decisions have been arrived at by trial and error, while some decisions, such as the use of FOSS, have been a cornerstone of our efforts. The next section explains a procedure we use to train a large number of students through FOSS and spoken tutorials. The textbook companion section explains how we create low cost documentation for FOSS through textbook companions. We follow this with a section on lab migration that helps shift lab courses based on proprietary software to FOSS. After this, we explain how these activities lead to a pyramid structure. Finally, we discuss the relevance of the low cost computing device Aakash, and conclude. We emphasize the role of Python in all of the above.

\section{Approach and Design Decisions}

We started in 2009 the effort explained in this article, to promote FOSS, with the Indian Government support and funding [nm09]. Although there are many good FOSS systems, they are difficult to use because of lack of documentation and support. If we could bridge this gap, FOSS would become accessible to our students, who do not have access to good software otherwise, as many of them are proprietary in nature. We also did not want to promote commercial software with tax payer's money. Our funding agency [nm09] had also made a policy decision to exclusively use FOSS.

It does not mean that we wanted to erect a wall between us and those who use commercial software. We do work with students who only know how to use the MS Windows operating system, for example. We do accept tutorials that have been dubbed with Windows Movie Maker. We believe that by creating a good ecosystem, we can shift proprietary software users to equivalent FOSS systems. We have always wished to be an inclusive project.

We have been using simple technologies to create our instructional material, as these allow school going students also to participate in the creation effort. For example, we have eschewed the use of instructional methodologies that help embed interaction in our tutorials. In contrast, open source screen recording software, such as RecordMyDesktop [rmd], has limited features. Nevertheless, we have made the learning effective by developing strict processes in creation [guidelines] and use of Spoken Tutorials, such as, the side-by-side method (see Fig. 1) and SELF workshops, explained in the next section. 


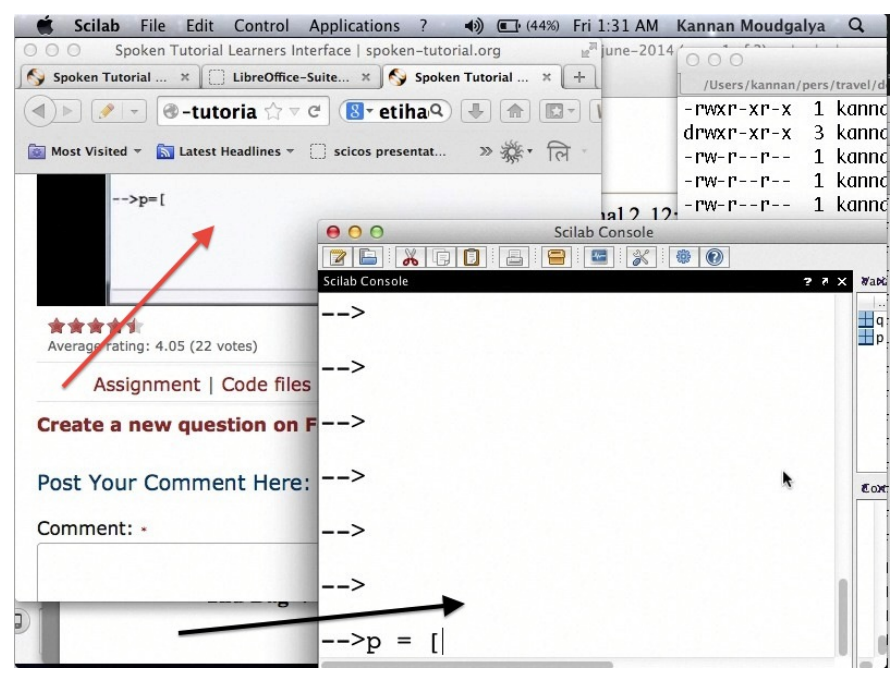

Fig. 1: A side by side arrangement for effective use of Spoken Tutorials. The red arrow points to the video tutorial and the black arrow points to the software studied, in this case, Scilab.

It is our policy that even "ordinary" people can participate in our project, using whatever equipment they have, and get recognised and also paid an honorarium. If an instructional tutorial is below professional quality, but reasonably good, we accept it. At the beginning of the project, we found that if we insisted on professional quality, it would necessitate the use of pin drop silence quality studios, established at a cost of millions of dollars. This would make the cost of spoken tutorials prohibitively expensive. Moreover, our project would become an elite one, filtering most "ordinary" people from participating in it.

We also wanted our methodology to be beginner friendly. When we started this work in 2009, most Forums that supported FOSS were quite unfriendly to the questions posed by beginners. For example, standard answers were,

- First learn how to post in the forum

- It is already answered, check the archives first

- Read the instruction manual first before asking a question

The reasons for the response given by the experts are understandable: there are only a few experts to answer the questions of large numbers of beginners, many of which may be repetitive. If the experts spend all their time in answering often repetitive questions, when will they have time to do their own work, develop the FOSS, remove bugs, write documents, etc., seem to be valid questions. Nevertheless, we can confidently say that only a small fraction of FOSS exploring/loving beginners stay with FOSS because of the above mentioned scoldings and the lack of support structure. The system we have built is beginner friendly, with enthusiastic participation from the experts.

When we started the project in 2009 , we decided to make our project a friendly interface between beginners and experts. One way to do this was through a Novice Check of the script, before creating a video tutorial. Unless a script is approved by a novice, it is not accepted for recording.

We illustrate the novice check with a bash script that the author reviewed as a beginner. The script asked the learner to download a bash file to the current directory and to type the name of the file on the console to execute it. On following the above instruction, the following error message appeared: Command not found. The

\begin{tabular}{lll}
\hline FOSS category & No. of Workshops & No. of Students \\
\hline C and C++ & 1,840 & 84,728 \\
Linux & 1,819 & 80,882 \\
PHP and MySQL & 997 & 44,414 \\
Scilab & 1,026 & 41,306 \\
Java & 672 & 31,795 \\
LaTeX & 771 & 30,807 \\
LibreOffice (all components) & 776 & 26,364 \\
Python & 419 & 18,863 \\
Total & 8,320 & 359,159 \\
\hline
\end{tabular}

TABLE 1: Total number of workshops conducted and the students trained in the past three years. The methodology is explained in the next section.

script writer forgot to state that there should be a ./ (dot-slash) before the file name, as the current directory is not in the path of beginner. After correcting this mistake, the same error message appeared. The reason for this is that this file is not executable. The script writer missed the following statement: the downloaded file should be made executable by the chmod command. These corrections were incorporated into the script before recording it.

Thus, a spoken tutorial is recorded only after the script, created by experts, is validated by beginners. After recording them, we run pilot workshops with the spoken tutorials. If there are minor difficulties, we mention the corrections in an instruction sheet. If there are major difficulties, the tutorials are re-created.

Although the details to be addressed in our tutorials seem to be excessive, the benefits are enormous. In Table 1, we give the total number of workshops that we have conducted and the number of students trained. The methodology developed by us to achieve such large numbers is explained in the next section.

An expert who knows that their tutorial will be watched 10,000 times will not mind spending a lot of effort to create outstanding instructional material. Insistence of passing through a novice check makes beginners important and also make them feel important. From the expert's point of view, once it is created, all beginners can be directed to see the tutorial. Finally, as we discuss next, the novice check and pilot workshops make our tutorials suitable for self learning, which in turn has resulted in large scale training, as demonstrated in Table 1.

The fact that a large number of people have undergone our LibreOffice workshops demonstrates that we are reaching out to the clerical staff and those who are at the doorsteps of IT literacy, and hence are at the bottom of the pyramid.

Our efforts to reach out to beginners has resulted in a pyramid structure: once the beginners are trained in a FOSS, they are encouraged to create textbook companions, to be explained below. Motivated students are then invited to participate in migrating lab courses to FOSS, and to use FOSS to create new software systems. Thus, bringing a large number of developers to our fold has the beneficial effect of producing a large number of FOSS developers as well. We begin with our training effort.

\section{Spoken Tutorial}

A Spoken Tutorial is an audio - video instructional material created for self learning through the Screencast technology. When this project started in 2009 , the main objective was to create documentation for FOSS, so that it is accessible to everyone. A 
detailed set of objectives and the method followed to achieve them are summarised in [kmm14].

We will begin with the reasons for calling this instructional material as a Spoken Tutorial. When this work started, there were a large number of silent Screencast tutorials on the Internet. To distinguish ours from these, we used the word spoken. This word is even more important, as we dub the spoken part into all Indian languages. As we do not capture the face of the person creating the tutorials, it is strictly not a video tutorial. Owing to the fact that one can use Spoken Tutorial to learn a topic, we call it a tutorial.

Spoken Tutorials have been released under a Creative Commons license and are freely downloadable from [Spoken]. There are about 500 original spoken tutorials in English and more than 2,000 dubbed tutorials in various Indian languages.

The Python Team created a set of 14 Spoken Tutorials on Python at the beginning. On using these tutorials, it was found that the pace of some tutorials was fast and that some topics were left out. A fresh set of 37 Spoken Tutorials have been created since then. These have also been dubbed into a few Indian languages.

At present, we have the following Python Spoken Tutorials at the basic level: 1) Getting started with IPython. 2) Using the plot command interactively. 3) Embellishing a plot. 4) Saving plots. 5) Multiple plots. 6) Additional features of IPython. 7) Loading data from files. 8) Plotting the data. 9) Other types of plots - this helps create scatter plot, pie and bar charts, for example. 10) Getting started with sage notebook. 11) Getting started with symbolics. 12) Using Sage. 13) Using sage to teach.

At the intermediate level, we have the following tutorials: 1) Getting started with lists. 2) Getting started with for. 3) Getting started with strings. 4) Getting started with files. 5) Parsing data. 6) Statistics. 7) Getting started with arrays. 8) Accessing parts of arrays. 9) Matrices. 10) Least square fit. 11) Basic data types and operators. 12) I O. 13) Conditionals. 14) Loops. 15) Manipulating lists. 16) Manipulating strings. 17) Getting started with tuples. 18) Dictionaries. 19) Sets.

At the advanced level, we have the following tutorials: 1) Getting started with functions. 2) Advanced features of functions. 3) Using Python modules. 4) Writing Python scripts. 5) Testing and debugging.

Spoken tutorials are created for self learning. The side-by-side method, a term defined in [kmm14]_ and illustrated in Fig. 1 is recommended for the effective use of spoken tutorials. This is a typical screen of the student running the tutorial. The learner is supposed to reproduce all the steps demonstrated in the tutorial. To achieve this, all supplementary material required for a tutorial are provided. We illustrate this with the Python Spoken Tutorial, loading data from files. In Fig. 2, in the command line, cat of the file primes.txt is demonstrated. By clicking the Code files link, shown with a red arrow, one may download the required files. In the figure, we have shown the window that pops up when the Code files link is clicked. This popped up window asserts the availability of the file prime.txt and also other files that are required for this tutorial. By clicking the link Video, also at the second last line of this figure, one can download the tutorial for offline use.

As these are created for self learning, and are freely downloadable, one should be able to learn from spoken tutorials directly from the website [Spoken]. Nevertheless, there are many reasons why we have been conducting organised workshops [kmm14] using spoken tutorials. As these are created for self learning, a domain expert is not required to explain the use of spoken tutorials

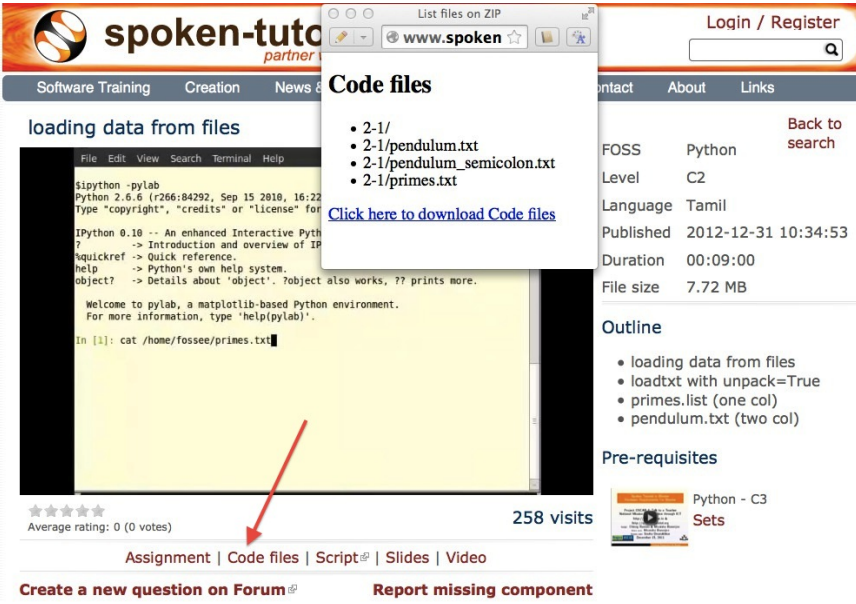

Fig. 2: Resources available for a spoken tutorial explained with an example. The file used in the tutorial is available through the Code files link, indicated by an arrow. On clicking this link, the available code files are displayed in a new window.

- a volunteer can organise these workshops. Based on trial and error, we have decided that our workshops should be of two hour duration and should be conducted as SELF workshops, as mentioned previously. Although these workshops are of only two hour duration, quite a bit can be learnt in a two hour workshop. For example, by no other method can a beginner learn LaTeX topics, such as compilation, letter writing, report writing, mathematical typesetting and introduction to beamer, in a two hour workshop [kmm11-TUGboat]. Although no domain experts may be available during these workshops, one may get one's questions answered through a specifically designed forum [forums].

Most students in India do not have access to good bandwidth and hence cannot access our web page. As a result, we need to provide the tutorials for offline use. In the previous paragraph, we have explained how to download a single video. To be consistent with our ethos, we have implemented a tool that allows the creation of an image consisting of many tutorials and downloading it for offline use. On choosing at [Spoken], Software Training > Download Tutorials $>$ Create your own disk image, one reaches the page shown in Fig. 3. Through this shopping cart like facility, we can create an image consisting of different FOSS families of spoken tutorials, in languages of one's choice. In this figure, one can see that the Python spoken tutorials in English and Tamil have been selected and these will take up about $680 \mathrm{MB}$. One may add many more FOSS categories, in one or more languages to the Selected Items list. Once all required tutorials are selected, one may click the Submit button. The image consisting of all the tutorials will be download as a zip file. On unzipping this file and opening the index.html file contained therein in a web browser, such as Firefox, all the selected videos can be played from the local drive. This zip file can be copied to all computer systems that are meant to be used in a workshop.

The Spoken Tutorial Team helps conduct SELF workshops [events-team]. The workshops are offered on about 20 topics, such as Python, Scilab, C, C++, Java, LibreOffice, LaTeX, PHP, Oscad and GNU/Linux. Organisers of SELF workshops at different institutions download the required spoken tutorials using the facility explained through Fig. 3, install the software to learn and ensure that the computer system, audio/video player and the headphone are in working condition. These organised workshops create a 


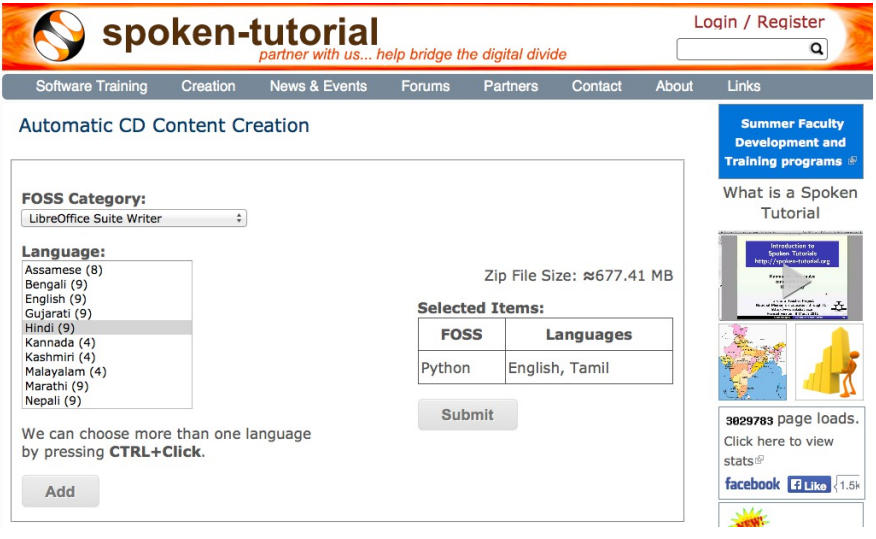

Fig. 3: The automatic CD content creation facility, available through [Spoken], by clicking Software Training > Download Tutorials > Create your own disk image. See that English and Tamil versions of Python tutorials are selected, with a size estimate of about $680 \mathrm{MB}$.

conducive ecosystem to learn through spoken tutorials.

As two hours may not be sufficient, one may not learn all the tutorials during a two hour workshop. After the workshop, the students are encouraged to download the tutorials and to practise by themselves at their home or office. The learners can post their difficulties, if any, on the Spoken Tutorial Forum [forums] based on the time line of a spoken tutorial. This special forum helps even beginners to locate previous discussions relating to spoken tutorials. An online exam is conducted a few weeks after the workshop and the participants who pass the exam are provided with certificates.

It is possible to get details of SELF workshops conducted by our team. In [python-ws-info], one can see summary details of the Python workshops that have taken place in the state of Gujarat. One can reach this information on [Spoken] by clicking the map of India, choosing Gujarat and sorting the result by FOSS. A screenshot is given in Fig. 4. In this figure, we have shown a red rectangle around a particular workshop that took place in Surat on 12 July 2013. By clicking the lens symbol, one can see the details of where the workshop took place, who conducted this workshop and so on. When the number of students who attended this workshop is shown in red (in this case, it is 51), it means that some of them have given their feedback. By clicking the number in red, one may locate the feedback given by students. A typical feedback is shown in Fig. 5.

We present some statistics of the people who have undergone Python SELF workshops. The number of SELF workshops conducted until now is 417, training close to 19,000 students, with 9,300 of them being females. It is interesting because it is believed that generally females do not take up programming in large numbers. Some of the reasons for this could be that they also find our methodology appealing, they are equally interested in employment, etc. Python SELF workshops have taken place in 23 states of India. Year wise break up of workshops is given in Table 2.

It should be pointed out that less than one half of the year is over in 2014.

The Python SELF workshops are effective. We have the following testimonials:

Through this workshop one can easily understand the basics of Python, which in turn can develop an

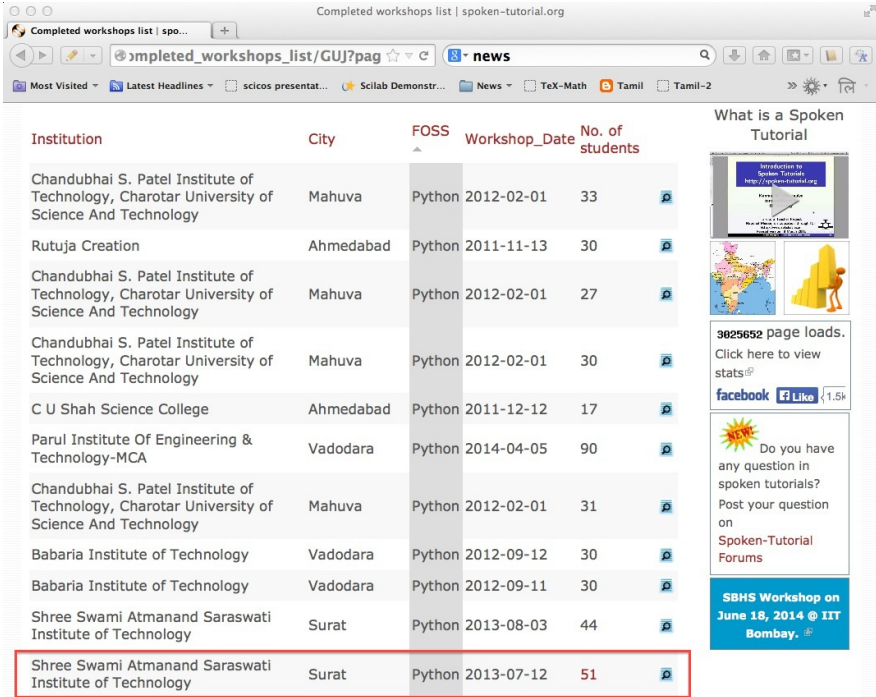

Fig. 4: Summary of Python workshops, obtained by clicking the India map in [Spoken], choosing Gujarat and then sorting by FOSS.

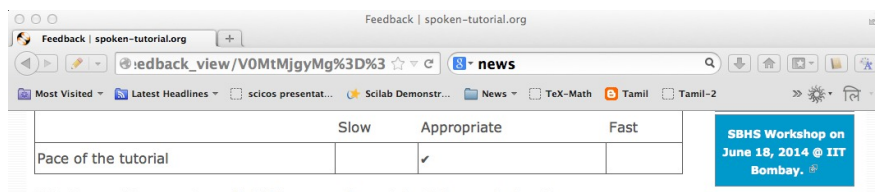

What was the most useful thing you learnt in this workshop?

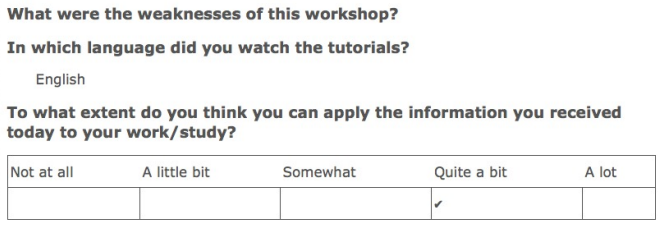

If so, how do you plan to use the information?

How would you rate the workshop on the following?

\begin{tabular}{|l|l|l|l|l|}
\hline \multicolumn{3}{|c|}{ Very low Below average } & \multicolumn{2}{c|}{ Average Above average Very high } \\
\hline Setup for learning & & & - & \\
\hline Computers in the lab & & & - & \\
\hline Audio quality & & & - & \\
\hline
\end{tabular}

Fig. 5: Feedback given by a student of Shree Swami Atmanand Saraswati Institute of Technology, Surat, Gujarat.

\begin{tabular}{lll}
\hline Year & No. of workshops & No. of students \\
\hline 2011 & 21 & 945 \\
2012 & 144 & 6,562 \\
2013 & 116 & 4,857 \\
2014 & 138 & 6,499 \\
Total & 419 & 18,863 \\
\hline
\end{tabular}

TABLE 2: Python SELF workshops, yearly statistics 


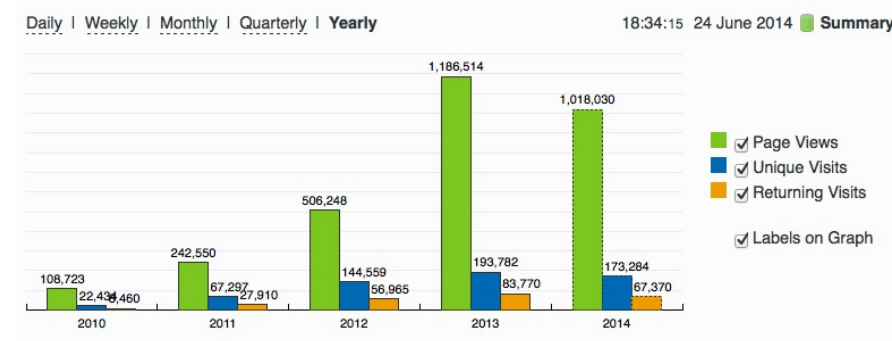

Fig. 6: Number of page views on [Spoken], since the beginning of this website. As there are many offline viewers in India, the effective number of page views may be considered to be at least twice these numbers.

interest in one's mind to learn more about Python. Thank you very much for this wonderful workshop.

-Brindersingh - Guru Nanak Institute of Technology, West Bengal

Got the initiative of how to work on Python that makes the programming comparative easy. Apart from this, graphical representation of mathematical formulation is quite good.

—Abhishek Bhargava - Institute of Engineering \& Technology, Alwar

Our website [Spoken] is becoming popular. Fig. 6 gives details of page views on our website. One can see that the number of page views are doubling every year. The number of people who benefit from our work is much higher than the numbers indicated in this figure. This is because, there are a lot of students in India who access our material through offline mechanisms, as explained earlier. For example, even though more than 80,000 students have undergone SELF workshops on Linux (Table 1), the largest number of server-recorded page views for any Linux video is only about 2,500 . It seems that the equivalent number of page views on our page is at least ten times the number indicated in Fig. 6.

A student who is trained through a SELF workshop is ready to contribute to the community. A textbook companion is the easiest way for them to contribute and in the process also get rewarded. This is explained in the next section.

\section{Textbook Companion}

One of the major shortcomings of FOSS tools is the lack of documentation. Proprietary software creators can deploy a lot of money and other resources to develop good documentation. We explain now how we have addressed this important issue through Textbook Companions.

We wanted to create documents for FOSS using India's abundantly available work force, namely, students. Unfortunately, creating a document requires a lot of experience in teaching. Students are good in writing programs, not documents. We explored the possibility of addressing this by solving the inverse problem: ask the students to write programs for existing documents. Textbooks can be considered as good documents. After doing a pilot with six students from different parts of India in the summer of 2010, we came up with the formula of one student, one month, one textbook companion.

Textbook companion (TBC) activity creates code for solved examples of standard textbooks using FOSS. These are created by students and the faculty of colleges from different parts of India. Students who create these books are given an honorarium of Rs. 10,000 for each companion. We were initially giving Rs. 5,000 honorarium to the teachers of these students for review and quality assurance. This has not worked well, as the teachers are generally not as knowledgeable and not as serious as the student who created the TBC. We have now shifted the review work to a general pool of experts, who are often students.

If anyone wants to understand what a program does, all that they have to do is to go through the corresponding example in the associated textbook. If TBCs are available for all textbooks used in educational programmes, students and teachers would not need proprietary software, at least for classroom use.

This programme is so flexible that almost anyone can contribute to the Python Textbook Companion (PTC) activity: from students to employees, teachers and freelancers. They can choose a textbook of their choice from engineering, science or social sciences, the only requirement being that Python is suitable for solving example problems. Upon successful completion of a PTC, the participant is awarded with a certificate and a handsome honorarium. PTCs are presented in the form of IPython Notebooks.

The PTC interface [PTC] displays all the completed books together with a screen-shot of code snippets, so that the user can easily download the PTC of their interest. The interface also allows the users to view all the codes of a chapter as an IPython notebook, which makes learning Python easy.

We use the following process to develop a PTC:

1) A student uploads Python code for the examples of one of the chapters of a chosen textbook. They should ensure that this book is not already completed nor under progress. They should also propose two other textbooks for PTC creation, in case the one they selected is already allocated to someone else.

2) Based on the Python code received for one chapter, our reviewers decide whether the student knows sufficient Python to complete the PTC. In case the selected textbook is already allocated to someone else, one of the other two chosen books is assigned. The student is given a time period of three months to complete the PTC.

3) The student has to upload the Python code in a specified format, on our portal.

4) Our reviewers check the correctness of the submitted code. They check whether the answers given by the code agree with those given in the textbooks.

5) Students who get all the code correct during the first review itself get a bonus, in addition to the honorarium mentioned above. Those who increase the work of reviewers by submitting wrong code are penalised and their honorarium gets reduced.

We currently have PTCs in the following categories: Fluid Mechanics, Chemical Engineering, Thermodynamics, Mechanical Engineering, Signal Processing, Digital Communications, Electrical Technology, Mathematics \& Pure Science, Analog Electronics, Computer Programming and others. Currently, there are $80 \mathrm{com}-$ pleted PTCs and 80 are in progress. PTCs so created are available for free download at [PTC].

The creators of PTC learn Python in a practical and effective way. One may see below testimonials from a few of the participants: 
I experienced that even an inexperienced person can do coding/programming. I gradually got to advance my skills in Python as I approached further in it. I got the IIT-B certificate, plus I got paid a handsome amount of cheque after completion which was good enough for me at then. -- Amitesh Kumar

I learnt Python from Spoken-Tutorials available on the website. The Python TBC team also helped me a lot in starting my internship. Till now, I have completed 3 TBCs and now, I know pretty much about python. I plan to take this project forward and Python is really helping me shine my resume. -- Deepak Shakya

This internship provided me a perfect platform and environment to learn Python. It helped me to incorporate both my ideas and practical work skills to the best. Especially, those concepts of $\mathrm{C}$ which are not present in Python gave me an awesome experience. Moreover, experience gained from it will make me capable of facing and overcoming the upcoming challenges under its applications. -- Ramgopal Pandey

We would like to point out some of the processes we have followed in the creation of PTC. Initially we tried to use the Sprint route to create PTCs. This involved a few people jointly coding all the problems, including unsolved problems, of a given book in one sitting. Solving unsolved problems made the task difficult. A book could not be completed in one session and those who coded for a part of the textbook often did not follow up to complete the work. There was also no ownership of the activity as many people were involved in one book. In contrast, the Scilab group used the approach explained previously and found it to be more effective, and more productive: there are 377 completed Scilab TBC and 266 are in progress. As a result, the Python group also changed the strategy for the creation of PTCs and this has yielded good results, as explained above. We are also in the process of contacting all who created Scilab TBC urging them to take up the PTC work.

The FOSSEE Team at IIT Bombay [FOSSEE] supports the following well known FOSS systems: Python, Scilab, OpenFOAM, COIN-OR. It also supports the following FOSS systems developed at IIT Bombay: Oscad (a locally developed for Electronic Design Automation and an alternative to OrCAD), Sandhi (an alternative to LabVIEW) and OpenFormal. We are in the process of creating TBCs for all of these systems.

\section{Lab Migration}

Students who successfully complete textbook companions, discussed in the previous section, are ready to help their colleges participate in lab migration, to be explained now.

Most of the academic programmes in India have laboratory courses that expect the students to carry out about ten experiments in a semester, in as many lab sessions, each lasting about three hours. Providing FOSS code through textbook companions does not necessarily enforce its use. On the other hand, if a FOSS system were to be used in a lab course, because of its compulsory nature, the use of FOSS system gets into the main stream. Similarly, the use of proprietary software in lab courses perpetuates its use. So long as a proprietary software is used in a lab course, any number of FOSS textbook companions will not wean the students away from the former.

\begin{tabular}{ll}
\hline $\begin{array}{l}\text { Commercial } \\
\text { software }\end{array}$ & FOSS equivalent \\
Matlab & Scilab \\
ORCAD & Oscad \\
Fluent & OpenFOAM \\
AnyLogic, Arena & SimPy \\
Witness, ExtendSim & \\
Quest, FlexSIM & \\
LabVIEW & Sandhi \\
\hline
\end{tabular}

TABLE 3: Migration of commercial software based labs to FOSS based labs

The FOSSEE team helps migrate commercial software based labs to FOSS. Once a faculty member in a college wants to migrate a lab to FOSS, we ask them or others in our network to come up with the required code in an equivalent FOSS and pay an honorarium. This code is made available to the public. Our team carries out lab migration given in Table 3. The most successful of them is Matlab to Scilab lab migration [LM]. We have migrated about 25 labs from Matlab to Scilab and about 15 more are in progress. On other FOSS families, we have migrated only a few labs, but the interest is growing. Although its progress is slower than that of TBC, lab migration can have a profound and lasting impact in promoting FOSS.

There is an important difference between a TBC and lab migration. The former is for a standard textbook and its utility is of general value: it may be of use to many students at more than one institution. A TBC is considered useful whether it is used or not in any one particular college. In contrast, the problem statements of a lab could be specific to a particular institution. Because of this, if the institution that participates in lab migration does not use the FOSS code it creates, the effort may be wasted. We insist that lab migration should not be just on paper, but be put in practice. Naturally, the progress in lab migration is slower compared to the TBC effort.

\section{Completing the Pyramid Structure}

In this section, we explain how our FOSS efforts help result in a pyramid structure of trained students. We started with SELF workshop based training, progressed to TBC and then reached lab migration, with each stage having increased complexity, as explained in the previous sections. In this section, we explain how a few other higher level activities that we have undertaken help result in a pyramid structure.

The next complicated task we have recently undertaken is to help our students do full projects using the FOSS that we support. Here is a feedback from a student who completed his Master's thesis using Oscad:

With intensive cooperation and guidance on Oscad EDA tool, from all of You, I have completed the project on "Design and Performance Analysis of OTA based SNR Meter" successfully and also submitted the project report today. Sincere thanks to all of You. Oscad is really user friendly and also highly accurate which is the main reason for completion of the project so smoothly.

We at Mangalore Institute of Technology and Engineering have decided to use Oscad for two of the labs "Linear Integrated Circuits and Analog communication" 
and "Power Electronics" labs. Your support is very much needed mainly for power electronics lab. Hope you will provide it. Thanks a lot. -- Harish Bhat

The next task is to help improve the FOSS itself or to use the FOSS to create new software. Typically, existing FOSS tools are used to create new FOSS systems. Python turns out to be an excellent glue language. We have used Python extensively in the creation of Oscad [oscad-book], [oscad-lj]. We are using Python extensively, once again, in the creation of Sandhi, a FOSS alternative to LabVIEW. Sandhi is yet to be released to the public. We have been using Python also to create online evaluation software to administer post SELF workshop tests.

The next level in this progression is possibly entrepreneurship. It is next level, because, an entrepreneurship is a lot more difficult compared to being a programmer. We also hope that the entrepreneurs who would come out of our work would be good in programming at the minimum. We are exploring the feasibility of grooming potential entrepreneurs from the students whom we train. At present we train about 200,000 students a year through SELF workshops. We expect about $1 \%$ of them to be good, possibly as a result of our FOSS promotion efforts. If $10 \%$ of this $1 \%$ are interested in becoming entrepreneurs, we will have about 200 people to train. Initial enquiries convince us that many groups that want to promote entrepreneurship may be interested in working with our selection. We believe that we can generate an army of entrepreneurs. If we succeed in this endeavour, we would really have achieved a pyramid structure.

The benefits of our effort are also in a pyramid structure. At the lowest level, the learners get IT literacy. At the next level, we have students passing exams, because of our training material, see a testimonial:

In my college, one of the students in btech 3rd year 1 st sem was having a makeup exam and and he was looking for guidance in learning Java. We gave the spoken-tutorial CD material on Java, and gave explanation on the contents of the $\mathrm{CD}$. After the exam he came and told that the spoken tutorial CD on java helped him a lot and that he developed confidence in Java by going thru all the tutorials and doing the assignments. He also told that the video tutorials cleared most of his doubts on java and helped him in passing the makeup exam. -Prof. K. V. Nagarjuna, Sree Dattha Inst. of Engg. and Science

Then, there are several levels of employment, starting from routine IT work, all the way up to work in niche areas, with attractive salaries. Finally, there is a possibility of one starting one's own company.

\section{Aakash: World's lowest cost computing device}

The agency that funded our FOSS promotion projects has created several e-content resources. It has also provided large amounts of bandwidth to educational institutions. These two do not get utilised effectively if the students do not have an affordable access device. If a student does not have an access device, they cannot participate in some of our projects. This affects their learning, while simultaneously resulting in loss of possible honorarium income. Aakash is the result of our efforts to address this problem [mpsv13], [sp13].

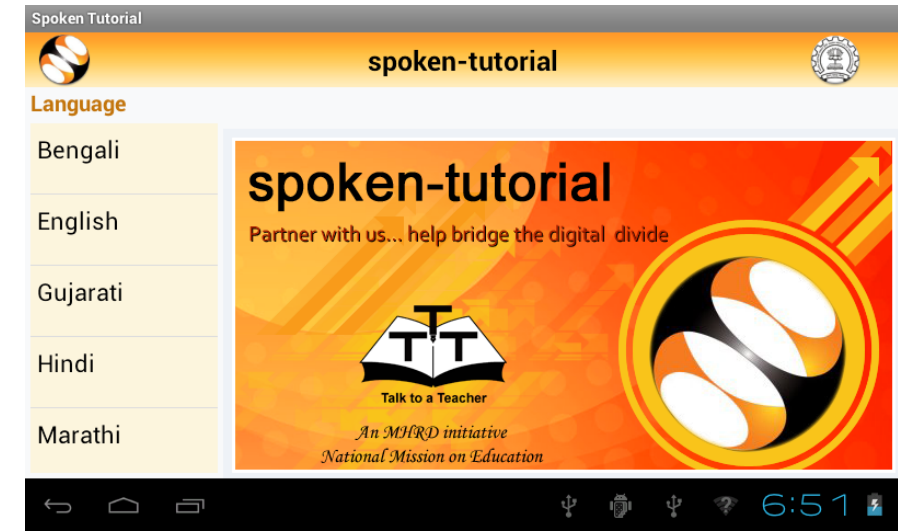

Fig. 7: Spoken Tutorials run on Aakash

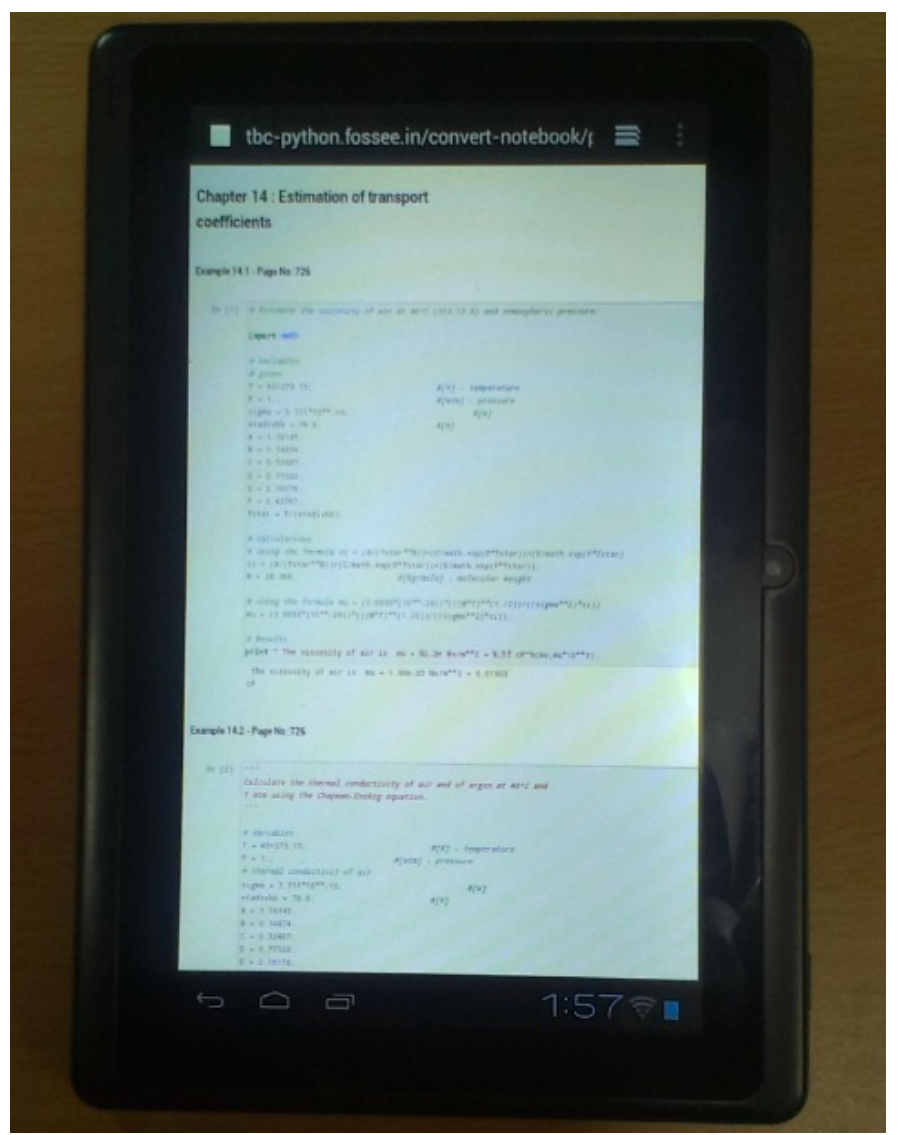

Fig. 8: A Python Textbook Companion on Aakash, the world's lowest cost computing device.

Aakash has indeed become a convergence device for our projects. Spoken Tutorials can be played on Aakash, see Fig. 7. A PTC running on Aakash is shown in Fig. 8.

\section{Conclusions and Future Work}

This article has summarised how the FOSS promotion work we started in 2009 has evolved into a massive training programme that caters to the bottom of the pyramid and also to those at the top. Given that our approach has worked for IT skills development, we are exploring the possibility of replicating this method to other skills development areas as well. It will be great if we can succeed in this, as India has a big shortage of skilled personnel and a large 


\begin{tabular}{|c|c|}
\hline IT & Information Technology \\
\hline FOSS & Free and open source software \\
\hline FOSSEE & $\begin{array}{l}\text { Free and open source software } \\
\text { for education }\end{array}$ \\
\hline PTC & Python Textbook Companion \\
\hline SELF & $\begin{array}{l}\text { Spoken Tutorial based Educa- } \\
\text { tion and Learning through free } \\
\text { FOSS study }\end{array}$ \\
\hline ST & Spoken Tutorial \\
\hline TBC & Textbook Companion \\
\hline
\end{tabular}

TABLE 4

number of youngsters who want employment. The training may have to start at school level and this is an order of magnitude larger problem. Finally, all our material and processes are in the open and are available to FOSS enthusiasts all over the world.

\section{Abbreviations \\ Acknowledgements}

The work reported in this article has been carried out by the $100+$ staff members of the FOSSEE and Spoken Tutorial teams. The author wishes to acknowledge the contributions of the Principal Investigators of these projects. The author wants to thank Prabhu Ramachandran for his help in converting this article to the required format.

\section{REFERENCES}

[events-team] Spoken Tutorial Project. Events team contact details. http://process.spoken-tutorial.org/index.php/SoftwareTraining\#Organising_Workshops, seen on 29 June 2014.

[forums] Spoken Tutorial Project. Online forum. http://forums. spoken-tutorial.org/, seen on 11 Feb. 2014.

[FOSSEE] FOSSEE Team. Free and open source software in education. http://fossee.in, Seen on 11 Feb. 2014.

[guidelines] Spoken Tutorial Team. Guidelines to create spoken tutorials. See http://process.spoken-tutorial.org/index.php/FOSS Stages/Checklist, seen on 11 Feb. 2014.

[kmm11-TUGboat] K. M. Moudgalya. LaTeX Training through Spoken Tutorials. TUGboat, 32(3):251-257, 2011.

[kmm14] K. M. Moudgalya. Pedagogical and organisational issues in the campaign for it literacy through spoken tutorials. In R. Huang, Kinshuk, and N.-S. Chen, editors, The new development of technology enhanced learning, chapter 13. Springer-Verlag, Berlin Heidelberg, 2014.

[LM] Scilab Team of FOSSEE. Matlab to Scilab lab migration. http://www.scilab.in/Lab_Migration_Project, Last seen on 5 July 2014.

[mpsv13] K. M. Moudgalya, D. B. Phatak, N. K. Sinha, and Pradeep Varma. Genesis of Aakash 2. CSI Communications, pages 21--23 and 29, Jan. 2013. Available at http://aakashlabs.org/ media/pubs/genesis-reprint.pdf, seen on 11 Feb. 2014.

[nm09] Ministry of Human Resource Development. National mission on education through ICT. http://www.sakshat.ac.in, Last seen on 11 Feb. 2014

[oscad-book] Y. Save, R Rakhi, N. D. Shambulingayya, R. M. Rokade, A. Srivastava, M. R. Das, L. Pereira, S. Patil, S. Patnaik, and K. M. Moudgalya. Oscad: An open source EDA tool for circuit design, simulation, analysis and PCB design. Shroff Publishers, Mumbai, 2013.

[oscad-lj] R. Rakhi and K. M. Moudgalya. Oscad: open source computer aided design tool. Linux Journal, pages 96-113, May 2014.

[PTC] Python Team of FOSSEE. Python textbook companion. http: //tbc-python.fossee.in, Seen on 19 June 2014. [python-ws-info] Spoken Tutorial Team. List of Python workshops in Gujarat. http://www.spoken-tutorial.org/completed_workshops_list/ GUJ? page $=42 \&$ sort $=$ asc $\&$ order $=$ FOSS, Last seen on 29 June 2014.

[rmd] recordMyDesktop Team., http://sourceforge.net/projects/ recordmydesktop/, Last seen on 27 June 2014.

[sp13] S. Patil and S. Patnaik. GNU/Linux on Aakash. CSI Communications, pages 28-31, July 2013. Available at http: //aakashlabs.org/media/pubs/GNU_Linux_on_Aakash.pdf.

[Spoken] Spoken Tutorial Project. Official web page. http://spokentutorial.org/, seen on 11 Feb. 2014. 